\title{
Efectos del cambio climático: Una mirada al Campo
}

\author{
Effects of climate change: A look to Agriculture
}

\section{Luis Enrique Arteaga N. ${ }^{1}$; Jairo Efrén Burbano N. ${ }^{2}$}

${ }^{1}$ Esp. Docente Investigador, Universidad Antonio Nariño, Pasto, Nariño, Colombia. luisearteaga@uan.edu.co.

2 Esp. Docente Investigador. Alcaldía de Pasto, Pasto, Nariño, Colombia. despacho@gestionambiental.gov.co.

Citar: Arteaga, L. \& Burbano, J. (2018). Efectos del cambio climático: Una mirada al Campo. Revista de Ciencias Agrícolas. 35(2): 79-91. doi:http://dx.doi.org/10.22267/rcia.183502.93

Recibido: junio 11 de 2017.

Aceptado: septiembre 13 de 2018.

\section{RESUMEN}

El cambio climático afecta de manera sensible al sector agropecuario, lo cual genera conflictos sociales, pérdidas económicas y pone en riesgo la seguridad alimentaria de la población; tal como se ha podido evidenciar durante los periodos de lluvias intensas y sequía presentados durante los fenómenos del niño y de la niña en Colombia. La solución a esta problemática requiere de procesos de planeación a largo plazo, basados en la recuperación de los ecosistemas, el uso de nuevas tecnologías y en la consolidación de una nueva identidad cultural orientada en la sostenibilidad, tal como se expresa en los planes y políticas de cambio climático del país. Esta revisión aborda los escenarios de cambio climático de Colombia y el departamento de Nariño, y evalúa los posibles impactos en el sector agropecuario; así como las diferentes acciones de mitigación y adaptación implementadas a nivel regional. Finalmente, se abordan algunas experiencias exitosas en América Latina como el cultivo de variedades resistentes al estrés climático, los sistemas agroforestales, la cosecha de agua y la lluvia sólida.

Palabras clave: adaptación, departamento de Nariño, mitigación, sector agropecuario.

\begin{abstract}
Climate change affects sensitively the agricultural sector, which generates social conflicts, economic losses and threatening food security; as evidenced during rainfalls and drought periods presented during the phenomena of "El niño" and "La niña" in Colombia. The solution to these problems requires
\end{abstract}


long-term planning processes, based on the recovery of ecosystems, the use of new technologies and the consolidation of a new cultural identity oriented towards sustainability; as expressed in the country's climate change plans and policies. This review addresses the scenarios of climate change in Colombia and the "Departamento de Nariño", and evaluates the possible impacts on the agricultural sector; as well as the different mitigation and adaptation actions implemented at the regional level. Finally, some successful experiences in Latin America are addressed, such as the cultivation of varieties resistant to climatic stress, agroforestry systems, water harvesting and solid rain.

Keywords: adaptation, departamento de Nariño, mitigation, agricultural sector.

\section{INTRODUCCIÓN}

La Convención Marco de las Naciones Unidas sobre el Cambio Climático (Naciones Unidas, 2015), en la conferencia de las partes realizada en Paris, reconoce al cambio climático como un problema global, que representa una amenaza urgente con efectos potencialmente irreversibles para las sociedades humanas y el planeta. En este sentido, afirma que para reducir los riesgos y efectos del cambio climático se requiere de la cooperación de todos los países, con el fin de lograr la reducción de las emisiones mundiales de gases de efecto invernadero. Así mismo, se propone mantener el aumento medio de la temperatura mundial por debajo de $2^{\circ} \mathrm{C}$ con respecto a los niveles preindustriales.

Esta afirmación reconoce al cambio climático como una consecuencia directa de la actividad humana, la cual ha alterado la composición de la atmósfera con efectos en la variabilidad natural del clima. Estudios realizados por el grupo intergubernamental de expertos sobre cambio climático IPCC, revelan el fuerte impacto que genera el cambio climático sobre los sistemas físicos y biológicos e influyen sobre las zonas costeras, las zonas de cultivo, los humedales, los páramos y los nevados, entre otros. De igual forma, los cambios de temperatura, las fuertes lluvias y los prolongados periodos de sequía observados durante los fenómenos del niño y de la niña, afectan el comportamiento de los ecosistemas, provocan el desplazamiento y extinción de las especies (IPCC, 2014).
Los efectos del cambio climático también se pueden apreciar a nivel socioeconómico, donde se evidencia la alta vulnerabilidad de las comunidades frente a las amenazas contra la salud pública, la disponibilidad el recurso hídrico, la seguridad alimentaria y la productividad industrial, entre muchos otros. Todos estos factores impactan de manera significativa a la economía del país, por daños en la propiedad y la infraestructura, pérdida de cosechas, costos de seguridad social, desempleo, migración de la población afectada, disminución del ingreso familiar y amenazas contra la seguridad. Para el caso de Colombia, se ha estimado un impacto negativo del cambio climático equivalente a una reducción anual de 0,49\% del PIB (Calderón et al., 2014).

Como se puede evidenciar en los escenarios de cambio climático propuestos por el IDEAM, el sector agropecuario del país no es ajeno a esta problemática. Según estos estudios, para el periodo 2010-2100, los sectores más afectados corresponderán a la ganadería, la agricultura y la pesca. Se estima que en promedio el sector ganadero tendrá pérdidas anuales en la producción de peso vivo de carne y leche de 1,6\%, mientras que en el sector agrícola se espera una reducción del 7,4\% en los rendimientos de los cultivos de maíz, arroz y papa. Finalmente, en el sector pesquero, se pronostica una disminución de la carga desembarcada del 5,3\%. Sin embargo, en el sector de la silvicultura se observa un efecto beneficioso, con un crecimiento del 6,2\% en el potencial forestal (DNP-BID, 2014). 
El presente estudio busca lograr una mejorar la comprensión de los efectos sociales, ambientales y económicos del cambio climático en el departamento de Nariño, a partir del análisis de los sectores de agricultura, ganadería, pesca y forestal. Así mismo, se analizan otros sectores que aportan de forma indirecta a la economía de la región; a través de la provisión de servicios ecosistémicos, como son los sectores de recurso hídrico y especies nativas. El análisis sectorial se orienta a responder las siguientes preguntas de investigación: ¿Cuáles son los escenarios del cambio climático? ¿Qué efectos produce el cambio climático en el sector agropecuario del departamento de Nariño? ¿Qué medidas de adaptación, mitigación y resiliencia se están implementando? ¿Qué otras acciones se pueden desarrollar para disminuir el impacto negativo del cambio climático?

\section{Escenarios de cambio climático}

Escenario de Cambio Climático en Colombia. Colombia ocupa el puesto 40 en emisión de gases efecto invernadero (GEI) en el mundo y el quinto lugar en América del sur, con producción del 0,36\% de las emisiones; sin embargo, sufre por igual los efectos del cambio climático $\mathrm{y}$, por lo tanto, debe impulsar estrategias de adaptación y mitigación con una visión de largo plazo. Del total de emisiones, el 52,8\% corresponde a la generación de energía, seguido por el 33,3\% debido a actividades del sector agropecuario. Las pérdidas por ineficiencia en los procesos representan el 8,3\% mientras que la industria ocupa el último lugar con el 5,6\% (Mengpin y Damassa, 2015).

Al comparar estos porcentajes con los promedios de los 5 países que más aportan a la producción de GEI, y de acuerdo con el plan energético nacional (UPME, 2015), se aprecia que la producción de energía ocupa un menor porcentaje; debido a que gran parte de la energía del país (67\%), se obtiene a partir de centrales hidroeléctricas. Esta dependencia, aumenta la vulnerabilidad en tiempos de sequía prolongados; donde la agricultura representa una fuente de energía alternativa, como es el caso del uso de la biomasa en centrales termoeléctricas o la elaboración de biocombustibles para el sector del transporte.

Desde un enfoque económico, el desarrollo del sector agropecuario representa una oportunidad para compensar el impacto negativo del menor crecimiento del sector minero energético, con reducción de la contaminación y aporte a la seguridad alimentaria. Finalmente, el elevado porcentaje de pérdidas observado que representa el 8,3\% de las emisiones, implica adelantar programas de eficiencia energética y reconversión tecnológica, orientados al uso de energías alternativas y a la sostenibilidad del sector productivo.

Según el DNP (2012), el cambio climático es considerado una amenaza para el logro de los objetivos del milenio, específicamente en lo referente a la erradicación de la pobreza extrema y el hambre provocadas por la reducción del acceso al agua potable; que impacta de manera negativa la productividad agrícola, con el consecuente incremento de los precios al consumidor. La Tabla 1 presenta una síntesis de los efectos esperados del cambio climático, causados por los eventos hidrometeorológicos extremos (Ortiz, 2012; DNP-BID, 2014; IPCC, 2014; IDEAM et al., 2015). 
Tabla 1. Impacto de eventos climáticos extremos en el sector agropecuario.

\section{Eventos extremos del cambio climático}

\section{Efectos en el sector agropecuario}

\begin{tabular}{|c|c|}
\hline $\begin{array}{l}\text { Aumento de las temperaturas } \\
\text { máximas y mínimas, olas de calor } \\
\text { y frío. }\end{array}$ & $\begin{array}{l}\text { - Aumento del estrés por calor y frío en los animales y especies } \\
\text { nativas. } \\
\text { - Aumento del riesgo de daño para los cultivos. }\end{array}$ \\
\hline $\begin{array}{l}\text { Eventos de precipitación más } \\
\text { intensos }\end{array}$ & $\begin{array}{l}\text { - Aumento en la erosión de los suelos. } \\
\text { - Daños a la infraestructura física } \\
\text { - Afectación a la conectividad vial por derrumbes. }\end{array}$ \\
\hline $\begin{array}{l}\text { Aumento de las sequías e } \\
\text { inundaciones asociadas con los } \\
\text { fenómenos del niño y de la niña. }\end{array}$ & $\begin{array}{l}\text { - Disminución de la productividad de los suelos agrícolas. } \\
\text { - } \text { Pesplazamiento de las zonas agroecológicas-productivas } \\
\text { - Dificultades para la alimentación del ganado } \\
\text { - Aumento de los precios al consumidor } \\
\text { - Disminución en la cantidad y calidad de las reservas acuíferas. } \\
\text { - Desabastecimiento de agua para riego. } \\
\text { - Aumento en el riesgo de incendios e inundaciones. } \\
\text { - Aumento de plagas y de enfermedades en los cultivos } \\
\text { - Incremento de los procesos de desertificación. } \\
\text { - Riesgos en la seguridad alimentaria. } \\
\text { - } \text { Deterioro de las condiciones de vida de los campesinos. } \\
\end{array}$ \\
\hline Aumento del nivel del mar & $\begin{array}{l}\text { - Inundaciones } \\
\text { - Erosión de los suelos y desaparición de la tierra arable } \\
\text { - Salinización de los suelos } \\
\text { - Disminución de la calidad del agua destinada a la agricultura } \\
\text { - Alteración de los patrones de distribución y migración de } \\
\text { peces y otras especies marinas. }\end{array}$ \\
\hline
\end{tabular}

Aumento de las temperaturas máximas y mínimas, olas de calor y frío.

nativas.

Aumento del riesgo de daño para los cultivos.
Debido a las sequías e inundaciones, el País ha sufrido impactos negativos sobre los ecosistemas, la biodiversidad y los sectores productivos (DNP, 2012). Durante el periodo de sequía provocado por el fenómeno del "El Niño", se evidenciaron problemas de disponibilidad de agua, incendios forestales y heladas; se afectó la producción de papa, cebada y trigo; así como en la producción pecuaria, principalmente la producción lechera (Pabón y Torres, 2007).

Las inundaciones generadas por el fenómeno de la niña 2010-2011, afectaron el 3\% de la superficie ganadera, equivalente a 1165413ha. Se estima que murieron 160965 animales orientados a la producción de carne o leche, con pérdidas de
\$108.652 millones. Los daños totales valorados en pecuaria, avicultura, acuicultura e infraestructura dentro de las fincas representaron un total de $\$ 759.893$ millones. Finalmente, la anegación de 1.000 .000 ha de cultivos y posterior marchitamiento y muerte de plantas, dejó un total de $\$ 693.448$ millones en pérdidas (BID et al., 2014).

Escenarios de cambio climático en el departamento de Nariño. Según el Plan de desarrollo departamental 2016-2019, en 2015 el fenómeno del niño afectó 12212 ha por incendios de la cobertura vegetal, 93117 ha por sequía, 6587 ha por heladas y 2558 ha por granizadas, que generaron pérdidas económicas cercanas a los 525 mil millones de pesos y afectaron a 41 de 
los 64 municipios que integran del departamento. Los sectores económicos de agricultura, ganadería y forestal, fueron particularmente sensibles a las modificaciones del clima, especialmente en los municipios ubicados en la zona andina (Gobernación de Nariño, 2016).

El impacto en el departamento es heterogéneo, evidenciando grandes pérdidas económicas, expresadas en miles de millones de pesos. Los municipios más afectados por las sequías son: Buesaco (20.270 ha), El Contadero (6.247 ha), San Bernardo (5.603 ha), La Cruz (5.238 ha), San Lorenzo (4.926 ha) y Albán (4.720 ha). El efecto de las heladas fue mayor en los municipios de: Potosí (11.247 ha), Ipiales (1.027 ha) y Córdoba (269 ha). Pupiales (21.9 ha) se vio afectado por las granizadas. Los municipios de Cumbal (3.300 ha), Guachucal (2.362 ha), Cuaspud (350 ha), Ipiales (320 ha) y Pupiales (176 ha) por las heladas (Gobernación de Nariño, 2016).

Los efectos del fenómeno del niño perjudicaron de manera negativa el $90 \%$ de los municipios del departamento con la pérdida gradual de la productividad, el aumento de la vulnerabilidad de los pequeños productores y el deterioro de las condiciones sociales. Estos efectos han sido superiores en las subregiones productoras de café, plátano, caña y papa. Así como en la región del altiplano, donde se afectó la producción de carne y leche, debido a la disminución de las precipitaciones en un 50\% ( $900 \mathrm{~mm}$ promedio anual); que afectaron 38947ha de pastos (CAF, 2000, DNP-BID, 2014). Así mismo, el sector avícola (13701 animales) y de cuyes (30020) se vieron afectados por los incendios presentados durante 2015.

En el departamento de Nariño, el cultivo de la papa constituye una actividad agrícola importante en las zonas frías. Se cultiva en 21 municipios, de los cuales se destacan Ipiales, Pasto, Pupiales, Túquerres, Sapuyes, Potosí, Guachucal, Aldana, Tangua, Cuaspud y Contadero, con un área cultivada de 23600 ha y una producción de 514020 toneladas al año (Consejo Nacional de la Papa, 2015). La papa es uno de los productos más sensibles a la variabilidad del clima, tanto por el incremento en las lluvias, como por sequías, heladas y granizo (García, 2010). El pacífico nariñense es una de las regiones más perjudicadas, especialmente en el sector de la pesca, dada su relevancia para el comercio y la provisión de alimentos. Esta situación se intensifica por las distintas presiones antrópicas ocasionadas por las actividades económicas legales e ilegales. En este sentido, se evidencia un modelo económico extractivista, sin participación de la población nativa, así como el uso inadecuado de los recursos naturales, particularmente con actividades como la minería y la tala indiscriminada de bosques (Gobernación de Nariño, 2016).

Estas problemáticas, se ven acentuadas por los mayores niveles de contaminación, y en consecuencia por el mayor número de emisiones de GEI, especialmente por los asociados con la creciente motorización y el crecimiento exponencial de la población en las ciudades intermedias y las regiones que hasta hace poco tenían vocación rural. Nariño presenta un incremento del 140\% del parque automotor de carros y del 108\% de motocicletas en el periodo 2010 a 2015 (Gobernación de Nariño, 2016). Este incremento tiene efectos negativos en la calidad de aire, los niveles de ruido y la movilidad, en detrimento de la salud y calidad de vida de la población. Según CORPONARIÑO (2014), el dejar de usar medios motorizados de transporte reduce la contaminación por fuentes móviles en un rango del 38 al 45\%.

Impactos futuros en el departamento de Nariño. Si los niveles de emisiones globales de GEI aumentan, como es lo más probable, la temperatura media anual en Colombia podría incrementarse gradualmente en $2,14^{\circ} \mathrm{C}$ para el año 2100 (IDEAM et al., 2015). Sin embargo, el aumento esperado en la temperatura, así como el comportamiento de las precipitaciones no será el mismo para todas las regiones de Colombia. Esto implica que las medidas para hacer frente a posibles fenómenos extremos deben ser diferentes para cada región del país.

Para el periodo 2071 - 2100, se estima que el departamento de Nariño tendrá un incremento de temperatura entre 1,9 y $2,3^{\circ} \mathrm{C}$. La zona pacífica la más afectada, con aumentos de $2,6^{\circ} \mathrm{C}$ sobre el valor de referencia actual, los cuales serán acompañados de 
un mayor nivel de precipitación, en el rango del $20 \%$ al 30\% (IDEAM et al., 2015). En la costa de Tumaco, se espera un aumento del nivel del mar cercano a los $40 \mathrm{~cm}$; con la consiguiente pérdida de terrenos, así como cambios en la salinidad del agua, por lo que se prevé emigraciones y migraciones inusuales de peces, que ponen en riesgo la seguridad alimentaria y los ingresos de la región pacífica (CORPONARIÑO, 2014).

Actualmente, el 51\% del departamento de Nariño presenta un clima cálido, el 18\% es templado, 15\% frío, 13,3\% muy frío y 2,2\% extremadamente frío. En el futuro se espera un cambio significativo de climas muy frío a frío, y frío a templado. Las categorías: desértico, árido y semiárido, de la cuenca del Río Patía presentarán un aumento en su nivel de precipitación, esperando un clima más húmedo. Las zonas con mayor humedad, como las ubicadas en los municipios de Mallama, Cumbal, Ricaurte, Santa Cruz, Sapuyes y Túquerres, cambiarán a climas más secos. Para el periodo de 2050 se espera un aumento de la humedad en la región norte y su disminución en la región andina (Guevara et al., 2016).

Según IDEAM et al. (2015), las principales consecuencias podrían verse en el sector agrícola, por el aumento de plagas y enfermedades en los cultivos de la región montañosa. El sector vial podría verse perjudicado por el incremento de derrumbes, los cuales también afectarán a las comunidades asentadas en zonas con pendientes elevadas. El sector ganadero será impactado por el aumento de la temperatura; que ocasiona estrés térmico y menor disponibilidad de pastos. La biodiversidad asociada al piedemonte costero podría presentar desplazamientos altitudinales por estrés térmico (IDEAM et al., 2015). Se prevé que el $47 \%$ de la economía campesina de la zona andina, podrían recibir un impacto alto y muy alto por la reducción de las lluvias en el periodo de 2011 a 2040 (Gobernación de Nariño, 2016).

Según el estudio sobre los efectos de la variabilidad climática en la disponibilidad de alimentos para la seguridad alimentaria en Colombia, los indicadores de sensibilidad y vulnerabilidad indican que el departamento de Nariño muestra distribuciones de ingreso con altos porcentajes de personas en riesgo de presentar condiciones de pobreza o indigencia; ante una caída del ingreso real asociado al aumento en el nivel de precios de alimentos, por lo cual, es considerado un departamento prioritario en esta materia. Los municipios con mayor riesgo se ubican en Pasto, Nariño, La Tola e Ipiales (Alfonso y Alonso, 2012).

\section{Adaptación, mitigación y resiliencia}

\section{Medidas implementadas en el departamento de} Nariño. Frente a los efectos observados y futuros del cambio climático, la administración departamental ha apoyado medidas ambientales en la prevención, protección y conservación de los ecosistemas estratégicos de este territorio. En este sentido, se adelantan procesos de conservación de las cuencas de los ríos Mayo, Guáitara y Juanambú mediante la gestión de proyectos estratégicos financiados con el Sistema General de Regalías y recursos propios, orientados a recuperar 121585 ha de áreas de recarga hídrica, de acueductos municipales y de 2077ha a través de procesos de compra de predios y programas de restauración (Gobernación de Nariño, 2016).

Otras iniciativas incluyen acciones para desarrollar el ecoturismo como una alternativa económica y de conservación de la diversidad biológica y cultural. Implementar planes de aprovechamiento sostenible del bosque natural y las especies de flora y fauna nativas, impulsar y fortalecer el uso y manejo sostenible de especies con potencial artesanal, promover el uso y manejo sostenible de especies de fauna silvestres para seguridad alimentaria y generar mecanismos de mercadeo y certificación para el uso sostenible de la biodiversidad (Delgado et al., 2008). Estas medidas se apoyan en instrumentos de planificación a largo plazo como el Plan de Gestión Ambiental de Nariño PEGAR 2015-2032 (CORPONARIÑO, 2014) y el Plan Territorial de Adaptación Climática del departamento de Nariño (Guevara et al., 2016). Las acciones estratégicas propuestas en ambos planes se relacionan en la Tabla 2. 
Tabla 2. Acciones a largo plazo propuestas en los planes de Gestión Ambiental PEGAR 2015-2032 y en el Plan Territorial de Adaptación Climática del departamento de Nariño.

\begin{tabular}{|c|c|}
\hline Plan & Acciones \\
\hline $\begin{array}{l}\text { Plan de Gestión } \\
\text { Ambiental } \\
\text { Regional de } \\
\text { Nariño PGAR } \\
\text { 2015-2032. } \\
\text { (CORPONARIÑo, } \\
\text { 2014). }\end{array}$ & $\begin{array}{l}\text { - Implementar estrategias de conservación para la protección de los ecosistemas; como la } \\
\text { - Inteclaratoria de áreas protegidas a nivel municipal. } \\
\text { - hídricas, cercas vivas y diversificación de parcelas agroforestales. } \\
\text { - Implementar las políticas de desarrollo limpio con el sector privado. } \\
\text { - Realizar una gestiónintegral del riesgo, especialmente en infraestructura yasentamientos. } \\
\text { - Mejorar la gestión integral del suelo para disminuir los procesos de erosión, aridización } \\
\text { y desertificación en el valle geográfico. } \\
\text { - Efectuar una gestión integral del agua, incluyendo la inundación y aumento de la } \\
\text { - } \text { escorrentía en las zonas costeras. } \\
\text { - Fesarrollar variedades agrícolas en las nuevas condiciones ambientales. } \\
\text { humedales, páramos y otras zonas de interés ecológico; dentro del plan de ordenamiento } \\
\text { - territorial. } \\
\text { - Implementar planes de continencia de enfermedades causadas por vectores, (dengue y } \\
\text { malaria) en la zona andina. } \\
\text { Fortalecer una cultura adaptativa; basada en el conocimiento ancestral de los grupos } \\
\text { étnicos sobre el uso sostenible de los recursos naturales. }\end{array}$ \\
\hline $\begin{array}{l}\text { Plan Territorial } \\
\text { de Adaptación } \\
\text { Climática del } \\
\text { departamento de } \\
\text { Nariño (Guevara } \\
\text { et al., 2016). }\end{array}$ & $\begin{array}{l}\text { - Identificar las variedades de cultivos con mejores características de adaptación a las } \\
\text { - } \text { condiciones locales. } \\
\text { - } \text { prodizar la planificación integrada de la producción que estimule la: "transformación } \\
\text { - Priorizar las medidas de protección de cultivos contra heladas y sequías. } \\
\text { - Modernizar y construir sistemas de riego para el suministro de agua a las explotaciones } \\
\text { agrícolas, realizando la sustitución gradual de los riegos de superficie. } \\
\text { - Conformar distritos de riego en las áreas geográficas que presentan condiciones de } \\
\text { riesgos climáticos sobre el recurso hídrico. } \\
\text { - Mejorar las unidades productivas agrícolas a partir de intervenciones en infraestructura } \\
\text { como: pozos, canales, tuberías, plantas de bombeo y derivaciones. } \\
\text { - Identificar e implementar prácticas como: procesos silvopastoriles, el uso de residuos } \\
\text { para el compostaje, y el uso de cultivos perennes; que contribuyan a la reducción de } \\
\text { presiones sobre el recurso hídrico y la conservación de la biodiversidad. } \\
\text { - Generar información climática a una escala relevante para la toma de decisiones; esto } \\
\text { incluye, por ejemplo, ampliar la red de estaciones meteorológicas en zonas aptas para } \\
\text { cultivos priorizados. } \\
\text { - Profundizar en los análisis de riesgos climáticos del sector agrícola, a través de la } \\
\text { formación de los involucrados en las cadenas de valor de los productos agrícolas. } \\
\text { - Fortalecer cadenas de producción y consumo responsables, promoviendo una cultura } \\
\text { emprendedora sostenible que apoye la transición hacia una economía con bajo contenido } \\
\text { de carbono. } \\
\text { Desarrollar instrumentos como seguros agrícolas, tendientes a disminuir los riesgos } \\
\text { climáticos y el impacto en las condiciones económicas de este sector productivo. }\end{array}$ \\
\hline
\end{tabular}


Medidas implementadas en el municipio de Pasto. La alcaldía de Pasto ha incluido dentro del plan de desarrollo "Pasto Educado Constructor de Paz" 2016 - 2019, el Nuevo pacto por la Naturaleza, donde se incluye la ruta especializada de gestión ambiental (Alcaldía de Pasto, 2016). Los componentes de esta ruta incluyen acciones orientadas a la sostenibilidad ambiental (Tabla 3).

Tabla 3. Acciones a nivel municipal desarrolladas en el Plan de desarrollo "Pasto Educado Constructor de Paz" 2016 - 2019 dentro de la ruta especializada de gestión ambiental.

\begin{tabular}{|c|c|}
\hline Eje Estratégico & Acciones \\
\hline $\begin{array}{l}\text { Sostenibilidad } \\
\text { ambiental }\end{array}$ & 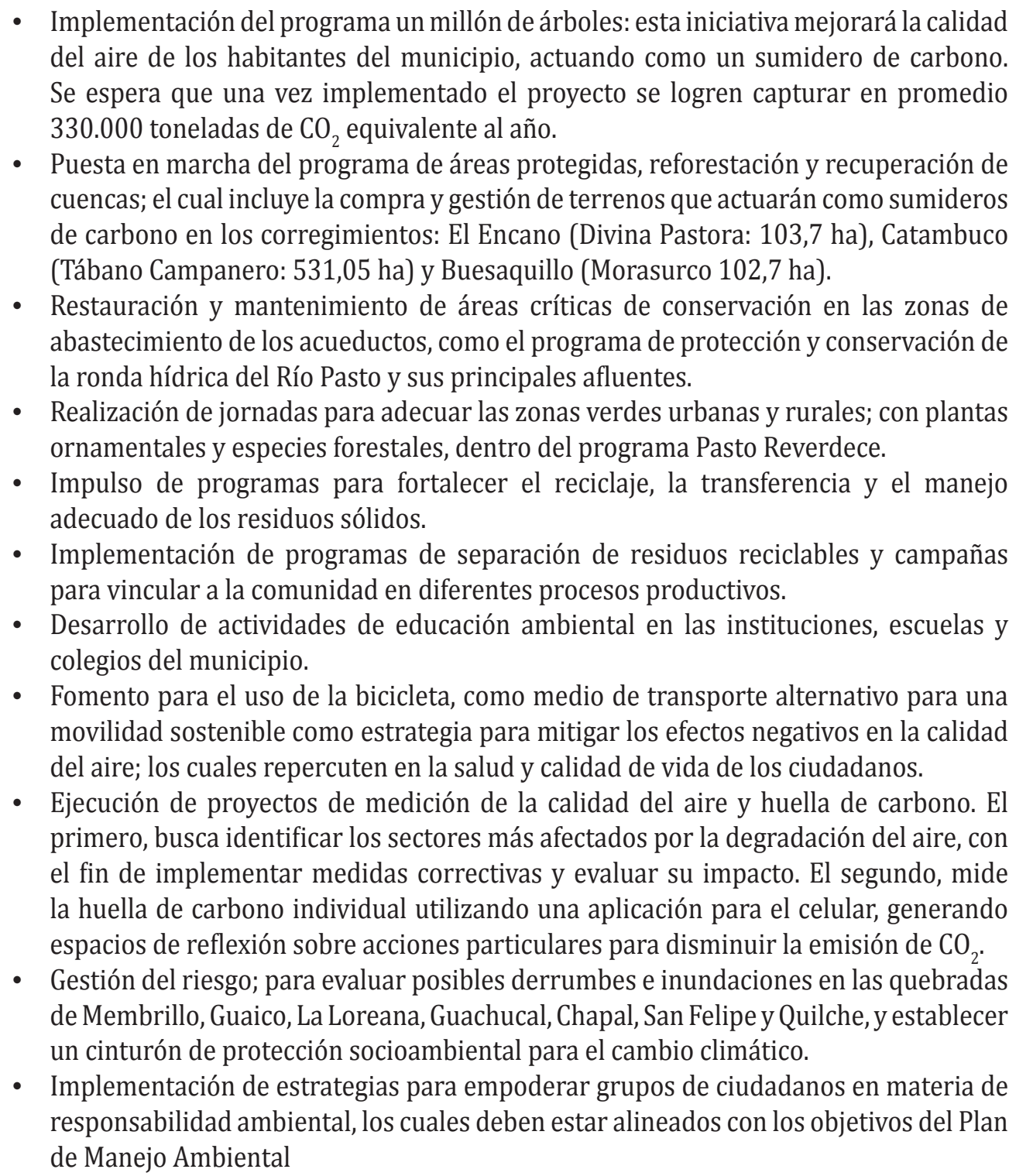 \\
\hline
\end{tabular}




\section{Acciones para reducir el impacto del cambio climático}

\begin{abstract}
Algunos cultivos y especies prometedoras. La biodiversidad es la clave para obtener variedades resistentes a estrés climático, aprovechando las especies locales con genotipos tolerantes a condiciones climáticas extremas; las cuales fueron desarrolladas por las comunidades indígenas como una estrategia de adaptación. Por ejemplo, en México, Perú, Bolivia y otras comunidades andinas, se han identificado cultivos de maíz, papa, ají, fríjol, maracuyá, habas, cebolla, ocas, ollucos, zanahoria y quinua, tolerantes a las sequías y lluvias prolongadas, así como con gran capacidad de adaptación a diferentes tipos de suelos (Torres y Gómez, 2008; García, 2010; Hellin et al., 2014). En el caso del departamento de Nariño, estas plantas representan un gran potencial de adaptación al cambio climático.
\end{abstract}

En los últimos años se ha difundido el uso de variedades silvestres como fuente de nuevos genes para sus parientes comerciales, con el fin de hacerlos más resistentes a la variedad del clima. El proyecto "Adaptando la Agricultura al Cambio Climático" es una iniciativa global para coleccionar y proteger la diversidad genética de plantas claves para la alimentación humana (Porch et al., 2013; Dempewolf et al., 2014). Como ejemplo se tiene el caso del cultivo de la soja en el Cerrado (Brasil), donde se desarrollaron variedades tolerantes a la variabilidad del clima que no requieren fertilización nitrogenada y aumentan la producción empleando menores recursos económicos (Ramalho et al., 2009).

Al igual que el sector agrícola, la ganadería contribuye al cambio climático, puesto que es un importante productor de GEI; y también se verá afectada por él. En conjunto, se estima que el sector ganadero genera el $37 \%$ de todas las emisiones de metano inducidas por el hombre, con un potencial de calentamiento global 23 veces superior al del dióxido de carbono (FA0, 2012). La variabilidad del clima, las olas de calor, el descenso de las precipitaciones, la degradación de las tierras, la falta de alimentos para los animales y la escasez de agua, influirán de forma directa e indirecta, en la producción y productividad del sector ganadero.

El aumento previsto del precio de los piensos, la energía y el agua, así como la mayor demanda de estos insumos para la elaboración de biocombustibles y otros usos industriales, afectarán la productividad del sector ganadero. Esta situación, sumada a la disminución de las tasas de reproducción y ganancia en peso del ganado, la menor producción de leche y la mayor frecuencia para la aparición de enfermedades infecciosas, requiere de razas adaptadas genéticamente a las modificaciones del entorno (Ortiz, 2012). Esta tendencia acrecentará la ventaja comparativa de los monogástricos, como aves y cerdos, que ofrecen una tasa más elevada de conversión del pienso que los rumiantes (Nelson et al., 2009).

El mejoramiento genético requiere del estudio de la velocidad de adaptación de las especies comerciales, para lo cual es necesario que los criadores de ganado cuenten con una amplia variedad de recursos genéticos; por lo cual surge la necesidad de introducir otras razas que presenten los caracteres deseados. Este es el caso de los camélidos andinos, los cuales son más resistentes a los abruptos cambios de los fenómenos naturales y proporcionan carne, fibra y piel. Nuevamente, para implementar estas estrategias se requiere del estudio de las tecnologías y sabidurías ancestrales, con un enfoque científico (Icuña, 2015).

Sistemas agroforestales sostenibles. Otro aspecto importante para adaptarse al cambio climático, es el mejor aprovechamiento del suelo y los recursos hídricos. En este sentido, ya existen estudios sobre la implementación de sistemas silvopastoriles, que combinan plantas forrajeras, arbustos y árboles; los cuales pueden aportar a la protección de las zonas de recarga hídrica y a la regulación hidrológica. Así mismo, estos sistemas pueden constituir una alternativa más eficiente y menos destructiva que la ganadería extensiva, puesto que 
permiten alcanzar niveles aceptables de productividad en condiciones climáticas adversas.

Los sistemas silvopastoriles proporcionan sombra, conservan la humedad del suelo y reducen la temperatura para el ganado. Además, pueden utilizarse para recuperar suelos de pastoreo degradado y como sumideros de carbono, así como corredores y refugio para la biodiversidad (Ortiz, 2012). Existe una amplia variedad de plantas que pueden utilizarse en este tipo de sistemas, según su función, pueden ser plantas para el consumo de los animales, como leucaena, pasto de guinea, botón de oro, matarratón, y pastos mejorados (Elvira et al., 2010; Uribe et al., 2011; Milera, 2011). También se pueden incluir variedades maderables como pino, eucalipto y el álamo (Luccerini et al., 2013); así como árboles frutales, como el mango, la guayaba, el limón y la naranja (Uribe et al., 2011).

Un árbol maderable, con potencial de industrialización y aprovechamiento en sistemas, agroforestales, corresponde a la Paulownia Tomentosa. Esta planta, de origen chino, es tolerante a suelos muy pobres o degradados por la erosión; resiste a la sequía, a las plagas y a las enfermedades y tiene un crecimiento acelerado, alcanza una altura entre 25 y $30 \mathrm{~m}$ en cinco años. Su madera puede ser usada para fabricar muebles, instrumentos musicales o como materia prima para la elaboración de papel. Así mismo, sus hojas de gran tamaño, son útiles como forraje para el ganado, purifican el aire, producen sombra abundante y retienen una cantidad importante de nitrógeno (Gutiérrez et al., 2015). Actualmente, en Colombia se están implementando éstos cultivos en el Valle del Cauca (Rivera, 2016); sin embargo, es necesario abordar estudios más rigurosos en este campo, para demostrar las ventajas de su cultivo en el país.

Cosecha del agua, reservorios y lluvia sólida. Asegurar la disponibilidad de agua y propiciar su uso eficiente, resulta imprescindible para enfrentar la variabilidad del clima en el sector agropecuario. Para ello, se han desarrollado diversas técnicas que incluyen las amunas, los diques, los atajados y la lluvia sólida. Las amunas, son sistemas peruanos para la recarga artificial de acuíferos, que utilizan zanjas abiertas para conducir el agua de la época de lluvia hasta cochas o reservorios, donde se infiltra en el subsuelo para surgir meses después durante la temporada seca (Alencastre, 2009). También se pueden construir diques con una cortina de tierra apisonada, que almacenan importantes volúmenes de agua en las zonas de ladera, la cual es utilizada para dar de beber al ganado y para el riego de las pasturas. Durante la construcción se debe acondicionar la cañería por debajo del terraplén, para suministrar el agua a través de bebederos, puesto que el acceso directo de los animales al reservorio contamina el agua de bebida (Duarte y Silveira, 2010).

Los atajados son reservorios Bolivianos que se construyen a partir de excavaciones, donde se recolecta el agua de escorrentía para usarla en momentos de escasez. Esta técnica permite reducir el riesgo de perder las cosechas en tiempo de sequía y crea un microclima local más húmedo y favorable para la vida silvestre (Goetter y Picht, 2014). Finalmente, la lluvia sólida es un desarrollo Mejicano, que almacena agua lluvia en forma de una sustancia polimérica de acrilatos de potasio súper absorbente. Es capaz de almacenar hasta 500 veces su peso. Como resultado se obtiene agua en pequeños pedazos, sin modificar su estructura química, que se puede almacenar en costales de fácil transporte. Este material no se pierde por filtración al subsuelo, por lo que resulta ideal para sembrar en zonas áridas, áreas de baja precipitación y en parcelas sin riego. Se han implementado experiencias exitosas en la siembra de maíz, tomate, girasol, papa y trigo (Rico, 2012).

\section{CONCLUSIONES}

Las lluvias prolongadas y los intensos periodos de sequía evidenciados durante los fenómenos del niño y la niña, impactaron la economía nacional y el sector agropecuario, especialmente a los pequeños productores del departamento 
de Nariño, donde se afectaron los cultivos de café, plátano, caña y papa, así como la producción de carne, leche y especies menores. Según el Plan de Desarrollo "Nariño Corazón del Mundo" (2016 2019), se estimaron pérdidas económicas cercanas a los 525 mil millones de pesos, donde se afectaron de manera negativa 41 de los 64 municipios que integran del departamento.

El cambio climático es un fenómeno global y creciente, que generará fuertes impactos socioeconómicos en el país, debidos principalmente a la variabilidad del clima. Este impacto será mayor en a las regiones más pobres, debido a su mayor vulnerabilidad frente a la seguridad alimentaria, la disponibilidad el recurso hídrico y la salud pública. Por esta razón, es responsabilidad del gobierno implementar estrategias de adaptación y mitigación a largo plazo; tal como se ve reflejado en los planes de desarrollo: nacional, departamental y municipal.

Se han desarrollado diferentes estudios para aprovechar la adaptabilidad y diversidad genética de cultivos andinos como: el maíz, la papa, el ají, el fríjol, las habas, la cebolla, las ocas, los ollucos y la quinua; al tiempo que se renueva el interés por las técnicas ancestrales de adaptación a la variedad del clima, como el caso de las amunas, los diques y los atajados. Estas alternativas, sumadas a los nuevos desarrollos tecnológicos, como la lluvia sólida y la creación de nuevas variedades vegetales y especies animales; tienen el potencial de beneficiar al departamento de Nariño. Es así como se presenta la oportunidad de aprovechar la biodiversidad del territorio, que sumada a la pluralidad cultural y étnica; permitirá afrontar de mejor manera los retos que impondrá el cambio climático.

Conflicto de intereses: Los autores declaran que no hay conflicto de interés.

\section{REFERENCIAS BIBLIOGRÁFICAS}

Alcaldía de Pasto. (2016). Acuerdo No. 12 Por el cual se adopta el Plan de Desarrollo del Municipio de Pasto 2016 - 2019 "Pasto Educado Constructor de Paz". Recuperado de https://www.pasto.gov.co/index.php/acuerdos/acuerdos2016?download=8829:acuerdo-012-plan-dedesarrollo-2016-2019.

Alencastre, A. (2009). Las amunas: recarga del acuífero en los andes. La gestión social del agua en Tupicocha, Huarochirí, Lima provincias. Cambio climático, crisis del agua y adaptación en las montañas andinas. Lima: Desco. 392p.

Alfonso, 0. \& Alonso, C. (2012). Estudio sobre los efectos de la variabilidad climática sobre la dimensión de la disponibilidad de alimentos en la seguridad alimentaria en Colombia e iniciativas de política. Recuperada de https://colaboracion.dnp.gov.co/CDT/Ambiente/ Resumen $\% 20$ Ejecutivo\%20Final\%20Pag\%20indiv.pdf

Banco Mundial. (2010). Informe sobre el desarrollo mundial. Washington: Banco Internacional de ReconstrucciónyFomento.Recuperadadehttp:// siteresources.worldbank.org/ INTWDR2010/ Resources /5287678-1226014527953/ Overview-Spanish.pdf

BID - Banco Interamericano de Desarrollo. CEPAL Comisión Económica para América Latina y el Caribe, DNP - Departamento Nacional de Planeación \& IDEAM - Instituto de Hidrología, Meteorología y Estudios Ambientales. (2014). Impactos económicos del cambio climático en Colombia. Colombia: Pereira editores. Recuperada de https://repositorio.cepal.org/ bitstream/handle/11362/37879/S1500268_es. pdf;jsessionid=D52316D278D7F437B91A606A 99B349E8? sequence $=1$

Calderón, S. Romero, G. Ordóñez, A., Álvarez, A., Ludeña, C., Sánchez, L., De Miguel, C. Martínez, K. \& Pereira, M. (2014). Impactos económicos del cambio climático en Colombia. Washington D.C: Banco Interamericano de desarrollo. Recuperada de repositorio.cepal.org/bitstream/11362/37879/1/S1500268_es.pdf 
Consejo Nacional de la Papa. (2015). Bullets regional papa Nariño. Recuperada de http://sioc. minagricultura.gov.co/index.php/art-iniciocadenapapa/?ide $=17$

CORPONARIÑO - Corporación Autónoma Regional Nariño. (2014). Plan de gestión ambiental regional del departamento de Nariño PGAR 2015 - 2032. Recuperada de http://corponarino.gov. co/expedientes/planeacion/pgar20152032/ diagnosticoPGAR20152032-parte1.pdf

Dempewolf, H., Eastwood, R., Guarino, L., Khoury, C., Müller, J. \& Toll, J. (2014). Adapting Agriculture to Climate Change: A Global Initiative to Collect, Conserve, and Use Crop Wild Relatives. Agroecology and Sustainable Food Systems. Recuperada de https://www.tandfonline.com/ doi/pdf/10.1080/21683565.2013.870629

Delgado, A., Ruiz S., Arévalo, L., Castillo, G. \& Viles N. (2008). Plan de Acción en Biodiversidad del departamento de Nariño 2006 - 2030. Colombia: Corponariño. Recuperada de http:// corponarino.gov.co/expedientes/intervencion/ biodiversidad/partel.pdf

DNP - Departamento Nacional de Planeación, (2012). Plan de adaptación al cambio climático. Bogotá: Imprenta Nacional de Colombia.

DNP - Departamento Nacional de Planeación, BID Banco Interamericano de Desarrollo. (2014). Impactos Económicos del Cambio Climático en Colombia - Síntesis. Bogotá: Imprenta Nacional de Colombia.

Duarte, E. \& Silveira, D. (2010). Fuentes de agua para el abrevadero del ganado. En plan agropecuario. Recuperada de https://www.planagropecuario. org.uy/web/magazine/view/id/143/title/ revista-n\%C2\%B0133-marzo-2010.html

FA0 - Food and Agriculture Organization. (2012). Consecuencias del cambio climático para la pesca y la acuicultura. Visión de conjunto del estado actual de los conocimientos científicos. Recuperada de http://www.fao.org/3/ai0994s.pdf
García, M. (2010). Cambio climático en el Altiplano boliviano y producción de quinua, percepciones y realidades. Recuperada de http://www. infoquinua.bo/fileponencias/ p_GARCIA $\% 20$ Magali $\% 20 \mathrm{Cambio} \% 20 \mathrm{Climatico} \% 20$ en $\% 20$ el\%20Altiplano\%20Boliviano\%20 corregido(ProdA).pdf

Goetter, J. \& Picht, H. (2014), Adaptación al Cambio Climático: Cosecha de Agua de Lluvia con "Atajados" en Bolivia. Bolivia: Agencia de cooperación Alemana GTZ. 5p. Recuperada de https://www.researchgate. net/publication/309464980_Adaptacion_al_ Cambio_Climatico_Cosecha_de_Agua_de_Lluvia_ con_Atajados_en_Bolivia

Gobernación de Nariño. (2016). Ordenanza No. 012, Por la cual se adopta el Plan de Desarrollo Departamental "Nariño Corazón del Mundo", para el periodo constitucional 2016 - 2019. Recuperado de http://xn--nario-rta.gov. co/inicio/files/PlanDesarrollo/Plan_De_ Desarrollo_Nario_corazn_Del_Mundo.pdf

Gutiérrez, J., Reyes, R., Medina, A., Niembro, C. \& Morfín, L. (2015). Caracterización nutricional de las hojas de Paulownia elongata en el periodo previo a su caída. Revista Iberoamericana de Ciencias. 2 (3). Recuperada de http://www.reibci.org/ publicados/2015/mayo/0900110.pdf

Guevara, 0., Abud, M., Trujillo, A., Suárez, C., Cuadros, L., López, C. \& Flórez, C. (2016). Plan Territorial de Adaptación Climática del departamento de Nariño. Colombia: Corponariño y WWFColombia. 154 pp.

Hellin, J., Beuchelt, T., Camacho, C., Badstue, L., Govaerts, B. Donnet, L. \& Riis-Jacobsen, J. (2014). An innovation systems approach to enhanced farmer adoption of climate-ready germplasm and agronomic practices. Washington D.C: International Food Policy Research Institute (IFPRI).

Icuña, S. (2015). Camélidos en los Andes de Bolivia y Cambio Climático. Revista de Investigaciones Altoandinas-Journal of High Andean Research. 17(3): 469-472. 
IDEAM - Instituto de Hidrología, Meteorología y Estudios Ambientales, PNUD - Programa de las Naciones Unidas para el Desarrollo, MADS Ministerio de Ambiente y Desarrollo Sostenible, DNP - Departamento Nacional de Planeación, Cancillería. (2015). Nuevos Escenarios de Cambio Climático para Colombia 2011-2100. Herramientas Científicas para la Toma de Decisiones - Enfoque Nacional - Departamental: Tercera Comunicación Nacional de Cambio Climático. Recuperada de http://documentacion. ideam.gov.co/openbiblio/bvirtual/ 022964/ documento_nacional_departamental.pdf

IPCC-Intergovernmental PanelonClimateChange.(2014). Cambio climático 2014, Impacto, adaptación y vulnerabilidad, Resumen para responsables de políticas. Suiza: IPCC. 40p. Recuperada de https:// www.ipcc.ch/pdf/assessment-report/ar5/wg2/ ar5_wgII_spm_es.pdf

Luccerini, S., Subovsky, E. \& Borodowski, E. (2013). Sistemas Silvopastoriles: una alternativa productiva para nuestro país. 8 ed. Argentina: Apuntes agroeconómicos - UBA. 7p.

Mengpin, J. \& Damassa, T. (2015). Infographic: What Do Your Country's Emissions Look Like? World Resources Institute. Recuperada de http:// www.wri.org/blog/2015/06/infographic-whatdo-your-countrys-emissions-look

Milera, M. (2011). Cambio climático, afectaciones y oportunidades para la ganadería en Cuba. Pastos y Forrajes. 34(2): 127-143.

Naciones Unidas. (2015). Convención marco de las naciones unidas sobre el cambio climático. 40 p. Recuperado de https://unfccc.int/resource/ docs/2015/cop21/spa/109s.pdf

Nelson, G., Rosegrant, M., Koo, J., Robertson, R., Sulser, T., Zhu, T., Ringler, C., Msangi, S., Palazzo, A., Batka, M., Magalhaes, M., Valmonte-Santos, R., Swing, M \& Lee, D. (2009). Cambio Climático. El impacto en la agricultura y los costos de adaptación. Washington, D.C: Instituto Internacional de Investigación sobre Políticas Alimentarias IFPRI. 30p. doi: 10.2499/0896295370.
Ortiz, R. (2012). El cambio climático y la producción agrícola. BID. Recuperado de http:// www.vipp.es/biblioteca/files/original/ e7a4a8a00d9ba9390d273d6dc1bb5666.pdf

Pabón, J. \& Torres, G. (2007). Impacto socioeconómico de los fenómenos El Niño y La Niña en la Sabana de Bogotá durante el siglo XX. Colombia: Cuadernos de geografía. Recuperada de http:// www.redalyc.org/pdf/2818/281821949008. pdf

Porch, T.G., Beaver, J.S., Debouck, D.G., Jackson, S., Kelly J.D., \& Dempewolf, H. (2013), Use of wild relatives and closely related species to adapt common bean to climate change. Agronomy. 3: 433-461. doi:10.3390/agronomy3020433

Ramalho, M., Simoneti da Silva, G. \& Dos Santos Dias, $L$ (2009), Genetic plant improvement and climate changes Crop Breeding and Applied Biotechnology. Brazilian Society of Plant Breeding. 9: 189-195.

Rico, S. (2012). Lluvia sólida. Secretaría de agricultura, ganadería, desarrollo rural, pesca y alimentación. Recuperada de http://infosiap.siap.gob.mx/ opt/foroexpectativas/ presentaciones2012/ rvelasco.pdf

Rivera, J. (2016). En Colombia ya se cultiva la Paulownia, el árbol más rentable del mundo. En: Colombia inn. Recuperada de http://colombia-inn.com. co/en-colombia-ya-se-cultiva-la-paulownia-elarbol-mas-rentable-del-mundo /

Torres, J. \& Gómez, A. (2008). Adaptación al cambio climático. De los fríos y los calores en los Andes. Lima, Perú: Soluciones Prácticas - ITDG. 152p.

UPME - Unidad de Planeación Minero Energética. (2015). Plan Energético Nacional. Colombia: Ideario Energético 2050. Bogotá: Ministerio de Minas y Energía. 184p.

Uribe, F., Zuluaga, A., Valencia, L., Murgueitio, E., Zapata, A., \& Solarte, L. (2011). Establecimiento y manejo de sistemas silvopastoriles. Manual 1, Proyecto Ganadería Colombiana Sostenible. Bogotá, Colombia: GEF, Banco Mundial, FEDEGAN, CIPAV, 